\title{
SNAI1 overexpression induces stemness and promotes ovarian cancer cell invasion and metastasis
}

\author{
ZHEN-YU LU ${ }^{1,2^{*}}$, RONG DONG ${ }^{3 *}$, DONG LI ${ }^{4 *}$, WEN-BO LI', \\ FENG-QIN XU ${ }^{1}$, YING GENG ${ }^{1}$ and YUN-SHAN ZHANG ${ }^{1}$ \\ ${ }^{1}$ Center for Reproductive Medicine, Tianjin Central Hospital of Obstetrics and Gynecology, Tianjin 300100; ${ }^{2}$ Union Stem \\ Cell and Gene Engineering Co., Ltd., Tianjin 300384; ${ }^{3}$ Tianjin Medical University, Tianjin 300070; ${ }^{4}$ Tianjin Medical \\ University General Hospital, Tianjin 300052; ${ }^{5}$ Tianjin Medical University Eye Centre, Tianjin 300384, P.R. China
}

Received November 17, 2011; Accepted January 9, 2012

DOI: $10.3892 /$ or.2012.1685

\begin{abstract}
Ovarian cancer is the fifth most common cancer among women worldwide. Detection of metastasis of ovarian cancer is crucial for diagnosis and prolongs the life of patients. This study focused on whether SNAI1 overexpression relates to invasion of ovarian cancer in vitro and in vivo. Invasion, colony formation and wound healing assays and flow cytometric analysis were performed to test the invasion and proliferation of SKOV3 ovarian cancer cells after transfection. The effect of SNAI1 on ovarian cancer in vivo was validated using a murine xenograft model. In vitro, SNAI1 upregulation led to an increased percent of CD133+ ${ }^{+}$SKOV3 cells and promoted SKOV3 cell invasion and proliferation. In vivo, the SNAI1 overexpression group showed the highest rate of tumor growth compared with SNAI2 and the control group (60 and 50\%, respectively). Our results show that SNAI1 expression induces an increase in the number of $\mathrm{CD}_{133^{+}}$cells, a change important for the epithelial to mesenchymal transition and the proliferation in ovarian cancer. It is suggested that SNAI1 may serve as a novel target for ovarian cancer prediction and therapy.
\end{abstract}

\section{Introduction}

Ovarian cancer is the most lethal gynecological malignancy in the world, and is the fifth most common cancer diagnosis in females. At present, as there is no method of early detection, the majority of patients present with peritoneal dissemination and distant metastasis at the time of diagnosis (1-3). With disseminated disease, treatment is often unsuccessful and overall survival is short. The identification of an invasion-

Correspondence to: Dr Yun-Shan Zhang, Center for Reproductive Medicine, Tianjin Central Hospital of Obstetrics and Gynecology, Tianjin 300100, P.R. China

E-mail: tjzys@hotmail.com

*Contributed equally

Key words: ovarian cancer, epithelial to mesenchymal transition, SNAI1, invasion, SKOV3 cells related molecule associated with the early and rapid spread of ovarian cancer is the current focus of many investigators (4-6).

Invasion and metastasis are biological hallmarks of malignancy (7). The molecular mechanism responsible for invasion and metastasis is a key area for investigation (8-10). A number of molecules related to tumor invasion and spread in ovarian cancer have been reported and include the following: Twist, E-cadherin and SNAI1. However, the molecular changes associated with acquisition of metastatic ability in ovarian cancer progression are poorly understood $(11,12)$. The epithelial to mesenchymal transition (EMT) has been described as an important mechanism promoting invasion and causing metastasis of cancer. EMT describes a series of events during which epithelial cells lose many of their epithelial characteristics and take on properties typical of mesenchymal cells, which require complex changes in cell architecture and behavior (11,13-15). The EMT is basically an embryonic trait through which cells adopt a phenotype more amenable to migration and invasion. Forced overexpression of SNAI1 in our present model demonstrated that SNAI1 incresed the percentage of CD133 ${ }^{+}$SKOV3 cells, an alteration important for the EMT and for cell proliferation. Our findings are the first evidence linking stemness to EMT and support a role of SNAI1 in metastasis.

\section{Materials and methods}

Cell culture and transfection. The ovarian cell line used in this study was SKOV3 (acquired from the American Type Culture Collection, Rockville, MD). These cells were cultured in Dulbecco's modified Eagle's medium (DMEM) supplemented with $10 \%$ fetal bovine serum (Invitrogen). The vectors were transfected into cells using PEI (cat\#23966; Polysciences Inc., Warrington, PA).

Expression plasmids. Full-length SNAI1 and SNAI2 cDNA was generated by normal human embryo total cDNA, and digested with XhoI/EcoRI and subcloned into pcDNA3.1 vectors. The resulting constructs were confirmed by DNA sequencing. The siRNA coding oligos against human SNAI1 and SNAI2 were designed and verified to be specific to SNAI1 and SNAI2. The SNAI1-siRNA-targeting sequence was AAGCTGAGCAAGATTCAGACC. The SNAI2-siRNA- 
Table I. Antibodies used in this study.

\begin{tabular}{lll}
\hline Antibodies & \multicolumn{1}{c}{ Company } & Product no. \\
\hline SNAI1 & Abcam & ab82846 \\
SNAI2 & Abcam & ab27568 \\
E-cadherin & Santa Cruz Biotechnology & sc-7870 \\
Vimentin & Abcam & ab20346 \\
CD133 & Miltenyi & 130080801 \\
\hline
\end{tabular}

targeting sequence was CAGGACCTCGCCGCTGCAGAC (siBcl-2 nucleotides 200-221) (16). The U6 promoter with the SNAI1-siRNA or SNAI2-siRNA insert was subcloned into pRNA-U6-Neo (Genscript, China). A non-silencing siRNA sequence (target sequence AATTCTCCGAACGTGTCACGT) was used as the negative control.

Invasion and wound healing assay. The cell migration assay was performed using Transwell cell culture inserts (Invitrogen). The transfected cells were maintained for $48 \mathrm{~h}$ and allowed to migrate for another $24 \mathrm{~h}$. The passed cells were stained with crystal violet solution and the absorbance at $595 \mathrm{~nm}$ was determined. In wound healing assays, cell motility was assessed by measuring the movement of cells into a scrape. The speed of wound closure was monitored after 12 and $24 \mathrm{~h}$ by measuring the ratio of the distance of the wound at $0 \mathrm{~h}$. Each experiment was performed in triplicate.

Colony formation assay. Control and transfected cells were seeded in a 6-well plate at a cell density of 1,000 cells/well. After two weeks, clones were fixed with methanol and stained with 2\% Giemsa solution (Merck) for $10 \mathrm{~min}$.

Flow cytometry analysis. Cells were fixed with $75 \%$ ethanol after treatment. An indirect labeling method was used to incubate primary and secondary antibodies (Table I). Red fluorescence used PE (FL2 channel), whereas green fluorescence used FITC (FL1 channel). C6 flow cytometry (Accuri) was used for determination.

Western blot analysis (WB). The whole cell lysates were resolved by SDS-PAGE and transferred onto polyvinylidene difluoride membranes (Millipore). Blots were blocked and incubated with the monoclonal antibody (Table I), followed by incubation with a secondary antibody (1:2000; Santa Cruz Biotechnology, Santa Cruz, CA). Blots were developed using an enhanced chemiluminescence detection kit (ECL; Amersham Pharmacia Biotech, Piscataway, NJ). For protein loading analyses, a monoclonal $\beta$-actin antibody (1:200; Santa Cruz Biotechnology) was used.

Murine xenograft model. Six-week-old female NIH BALB/cnull mice were housed in the animal facilities of the Tianjin Medical University as approved by the Institutional Animal Care and Use Committee. SKOV3 cells $\left(10^{7}\right.$ cells $\left./ \mathrm{ml}\right)$ were mixed with Matrigel (BD Biosciences) and subcutaneously injected into the backs of nude mice $(0.1 \mathrm{ml} / \mathrm{mouse})$. For 25 days, the mice were monitored and tumor sizes were measured daily using a caliper. After 25 days, the experiments were terminated because of the tendency of HSC-3 cells to become necrotic and form skin ulcers. After completing the observation, the mice were sacrificed. Tumors were harvested and stored at $-80^{\circ} \mathrm{C}$ for subsequent tests.

Statistical analysis. All data in the study were evaluated with SPSS11.5 (SPSS Inc., USA). Differences were considered significant at values of $\mathrm{P}<0.05$.

\section{Results}

SNAII upregulation leads to increased SKOV3 cell proliferation. Given the upregulated and knockdown cell models in SKOV3 cells, as well as the detection of dowregulated
A

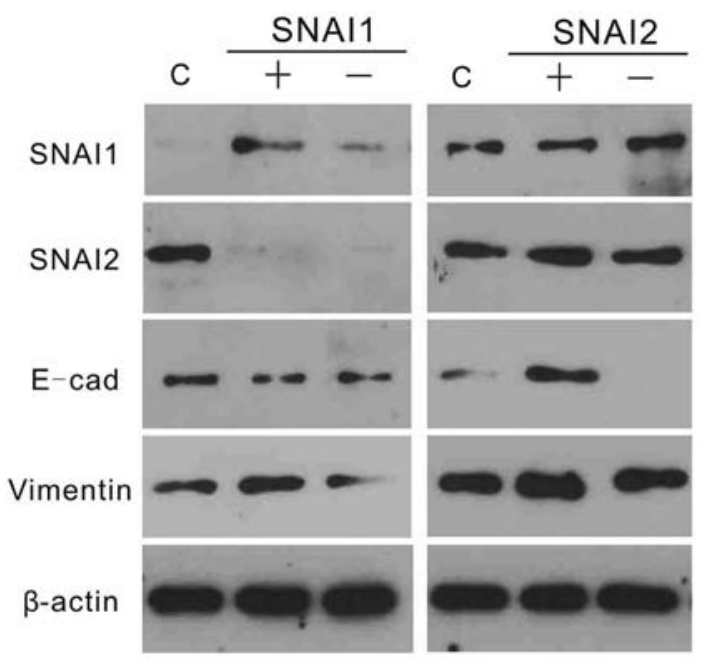

$\mathbf{B}$
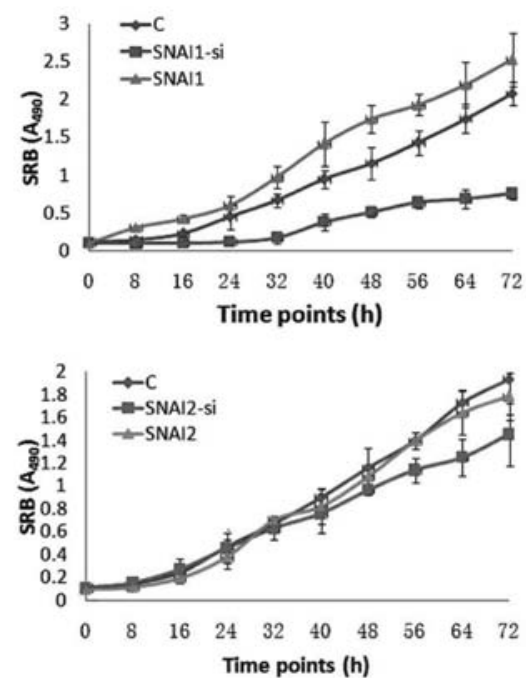

Figure 1. SNAI1 promotes SKOV3 cell proliferation. SKOV3 cells were trasfected with SNAI1 and SNAI2 expression plasmids or shRNA plasmid. (A) Western blot analysis (B) and the sulphorhodamine B (SRB) protein assay were performed to analyze the levels of invasiveness-related proteins and SKOV3 cell proliferation, respectively. 
A
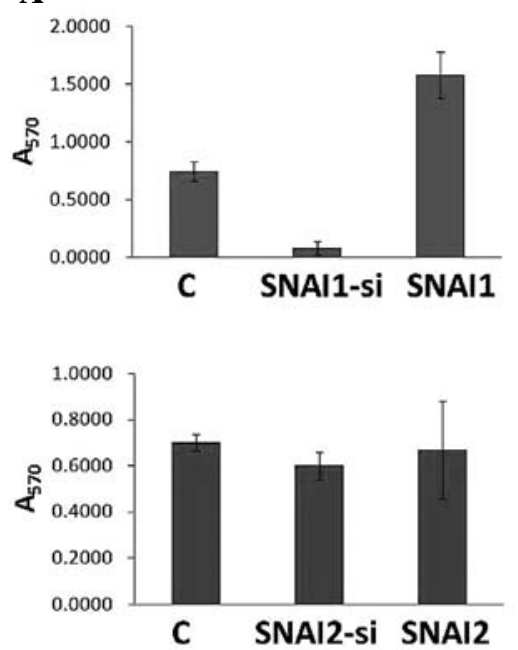

B
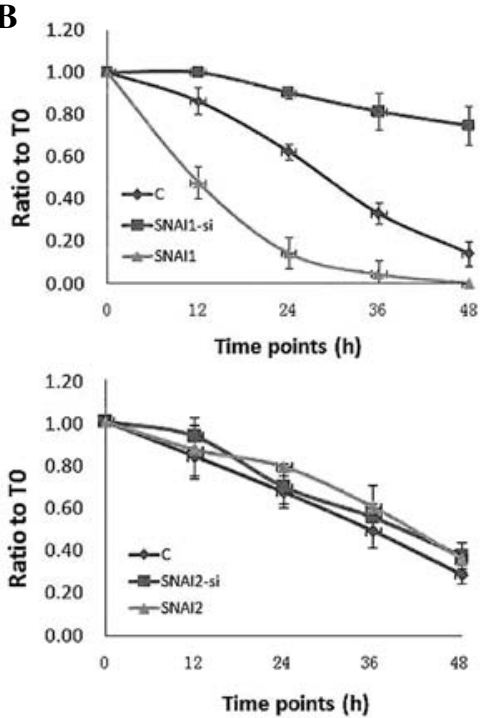

C

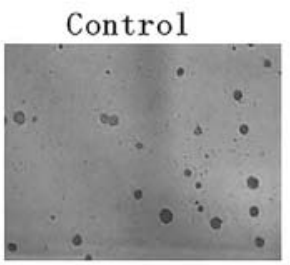

$\operatorname{SNAI1}(+)$
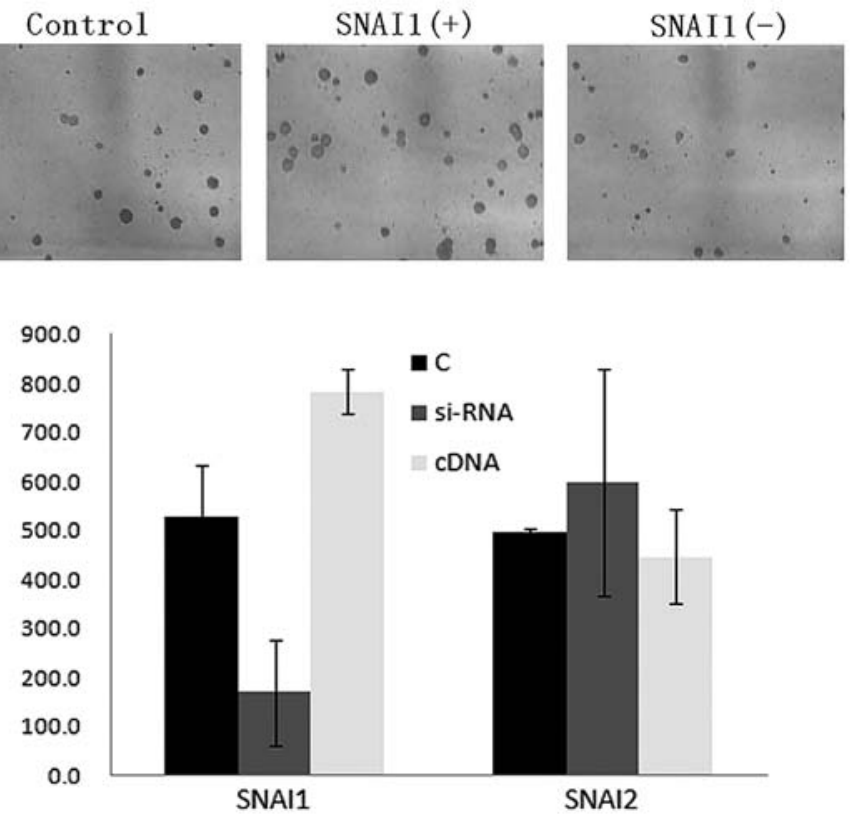

Figure 2. SNAI1 promotes SKOV3 cell invasion, migration, and clone formation. SKOV3 cells were trasfected with SNAI1 and SNAI2 expression plasmid or shRNA plasmid. (A and B) Wound healing and invasion assay was used to test the migration and invasive ability of SKOV3 cells. (C) In addition, the colony formation assay was performed to analysis the effect of SNAI1 and SNAI2 on clone formation of SKOV3 cells.

E-cadherin following the ectopic upregulation of SNAI1, we investigated the effect of SNAI1 activation on cell invasion, migration, and clone formation. E-cadherin is one of the most frequently reported characteristics of metastatic ovarian carcinoma. Metastasis is associated with cell migration and invasion, and occurs through a mechanism similar to that of EMT. We studied the invasion and migration ability of SKOV3 cells after performing SNAI1 and SNAI2 ectopic transfection and knockdown, respectively. As shown in Fig. 1A, following a western blot analysis, the quantitative analysis suggests a significant difference in the ectopic transfection groups compared to the control. SNAI1 and SNAI2 are upregulated in the overexpression groups, and are knocked down in the shRNA groups. In the sulphorhodamine B (SRB) protein proliferation assay, a significant difference in cell proliferation was observed following SNAI1 overexpression and knockdown in each of the groups examined (Fig. 1B). The cell line showed a significant decrease in cell proliferation following knockdown of SNAI1, and an increase due to SNAI1 overexpression to above control values $(\mathrm{P}<0.01)$. Importantly, no significant difference in the proliferation rate was noted between the groups when SNAI2 was overexpressed or knocked down $(\mathrm{P}>0.01)$.

SNAII upregulation leads to increased SKOV3 cell invasion, migration and clone formation in vitro. We then analyzed SKOV3 cultures for functional changes in migration, invasion and clone formation following transfection with SNAI1 and SNAI2, separately. Comparison of the transfection group with the control group, revealed a significant increase in the migration, invasion and clonogenicity in the SNAI1 overexpression group (Fig. 2). In order to rule out effects of non-uniform transfection efficiency, we selected for shRNA transfected cells 


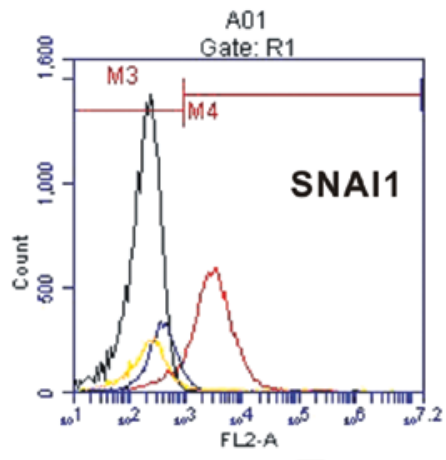

IgG control

Merch

$\operatorname{pcDNA}$

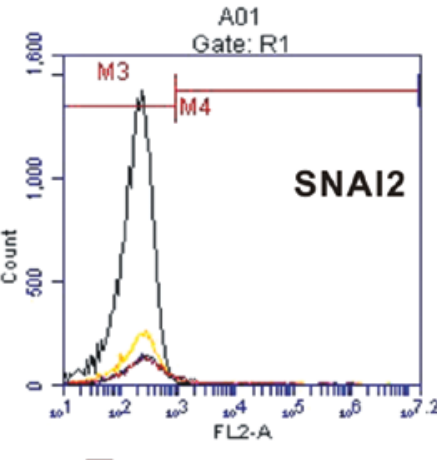

Figure 3. SNAI1 promoted percent of CD133 cells in SKOV3 cells. Flow cytometry analysis was performed to analysis CD133 ${ }^{+}$cell percentage in SKOV3 cells that trasfected with SNAI1 and SNAI2.

A

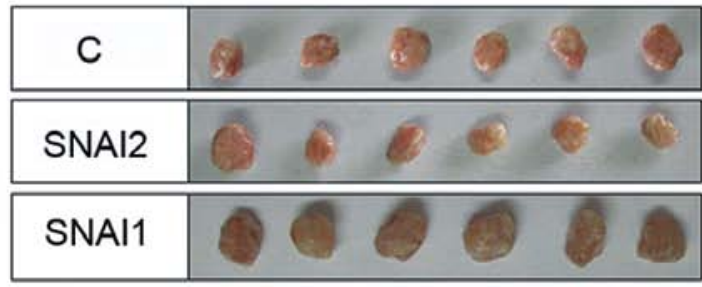

B

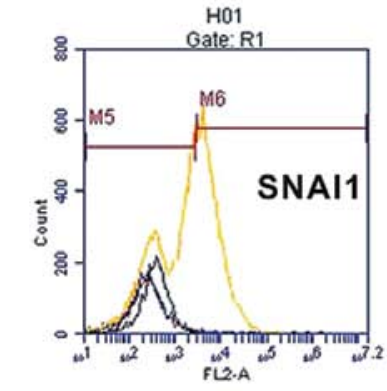

IgG control

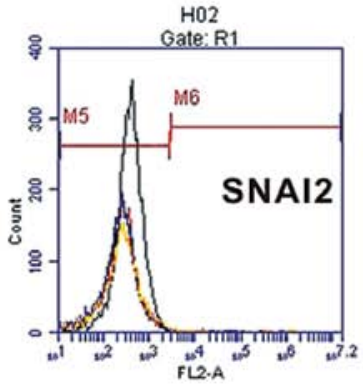

pcDNA $\square$ SNAI1 or SNAI2
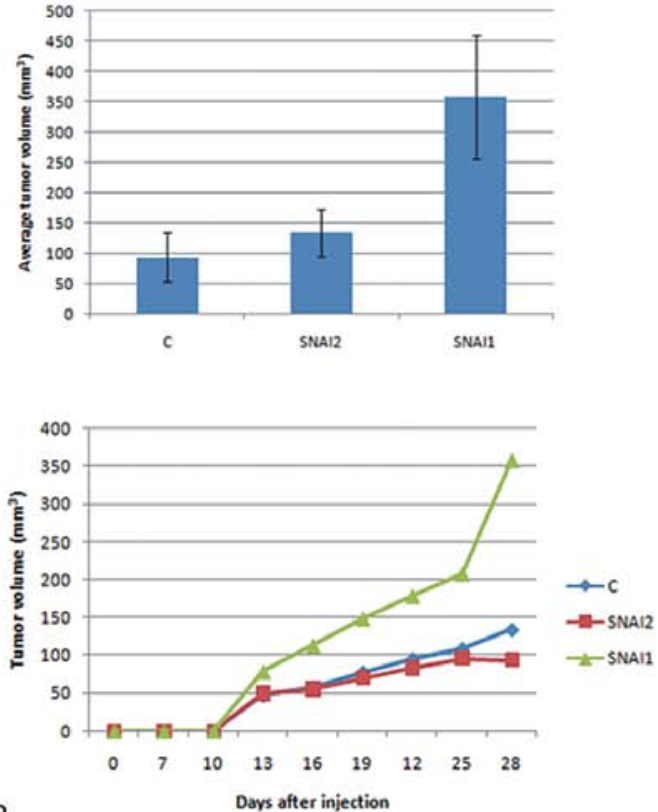

Figure 4. SNAI1 promote ovarian cancer proliferation in vivo SKOV3 cells overexpressed SNAI1 and SNAI2 were injected into nude mice. Then the tumor volume was recorded (A and $\mathrm{C}$ ) and $\mathrm{CD} 133^{+}$cell percentage was tested by (B) flow cytometry analysis.

with knock down of SNAI1, and observed decreased activities compared with control. Notably, SNAI2, the structural homologue of SNAI1, did not promote cell migration.

SNAII increases the percentage of $\mathrm{CD} 133^{+}$cells in SKOV3 cells. In the process of metastasis by tumor cells, stem cells play an important role in tumor cell plasiticity and proliferation, and are also strongly associated with the EMT. In this study, we examined the alterations in the percentage of $\mathrm{CD}_{133}{ }^{+}$cells present after overexpression and knock down of SNAI1 and the results were confirmed by flow cytometry. In the SNAI1 overexpression group, $\mathrm{CD}_{133}{ }^{+}$cells accounted for about $47.5 \%$. In the control transfection group, these cells accounted for $8.7 \%$, but for the knockdown group they accounted for only
$0.4 \%$ (Fig. 3). In the SNAI2 overexpression, knockdown and control groups, the percentage of $\mathrm{CD} 133^{+}$cells were not significantly different. Taken together, these observations suggest a synergism between SNAI1 and CD133 ${ }^{+}$cells which can result in increased proliferation, migration and invasion.

Co-expression of CD133 and SNAII correlates with tumor proliferation. To examine the in vivo effects of the expression of SNAI1 and SNAI2 on tumor development, a murine xenograft model was used. SKOV3 cells were utilized to establish xenografts in nude mice. Nodule formation and growth (volume) were monitored for 25 days. SNAI1 overexpression groups showed the highest rate of tumor growth compared with SNAI2 and the control group (60 and 50\%, respectively) (Fig. 4). We collected 
the cells from tumor tissues, and performed by flow cytometry analysis. The results demonstrated that the percentages of

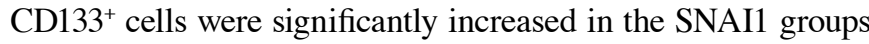
compared with controls, but no significant differences were observed between the SNAI2 groups and the controls (Fig. 4).

\section{Discussion}

Ovarian cancer as a silent killer, shows early extensive tumor invasion and peritoneal metastasis. Current therapies including anti-angiogenic therapies have not improved the survival rate ofpatients with ovarian carcinoma. The growth and metastasis of neoplasms depend, in part, on adequate stem cells support. The term 'EMT' has been used to reflect the embryonic-like ability of stemness in tumor cells (17-19).

EMT is a crucial process during embryonic development, but it has recently been found to play a role in neoplasia and fibrosis $(19,20)$. For epithelial cells to migrate into the tubulointerstitium, E-cadherin must be repressed. SNAI1 was the first transcriptional repressor of E-cadherin described, but subsequently two other Snail family members, including SNAI2 (Slug), have been identified as well as other non-Snail transcriptional repressors of E-cadherin (e.g., E47, _EF1/Zeb1, and Sip1/Zeb2). These repressors are tightly regulated at the transcriptional level and/or by subcellular localization. Insight into how EMT is regulated may provide new chemotherapeutic and antifibrotic therapies (21-23).

Tumor progression and invasion are complex biological processes that involve the remodeling of stromal tissue by invading cells (24). SNAI1-1 and SNAI-2 are members of an evolutionarily conserved family of zinc-finger transcription factors. Both are expressed in the intermediate mesoderm and the metanephric mesenchyme during renal development and are downregulated before epithelial differentiation $(16,25)$. Kidneys develop normally in mice with a loss-of-function mutation in SNAI2, suggesting a functional redundancy of SNAI-1 and SNAI-2. However, a recent report has shown that the E-cadherin repressors SNAI-1, SNAI-2, and E47 produce different genetic profiles when overexpressed in ovarian tumor cells, providing reason for differential regulation of these transcription factors (26-28). Our current results, showing that constitutive expression of SNAI1 leads to suppression of invasion through induction of the EMT, as well as the reciprocal results with gene knock down, are consistent with these previous studies. Our results also suggest that SNAI1 induces EMT and increases the percent of $\mathrm{CD} 133^{+}$cells, promoting the clonogenic ability of SKOV3 cells. SNAI1 activation induces stronger stemness and activates dormant developmental pathways in invading tumor cells. Therefore, suppression of invasion-related molecules, such as SNAI1 may be one mechanism by which SNAI1 suppresses metastasis. In this study, increased levels of SNAI2 have no significant effect on SKOV3 proliferation, migration, invasion and clonogenicity, also no effect on $\mathrm{CD}_{133^{+}}$cells.

Our results show that upregulation of SNAI1 expression increases the percent of $\mathrm{CD}_{133^{+}}$cells, an alteration important for the EMT and proliferation of ovarian cancer. SNAI1 may therefore represent a potential new target for preventing tumor invasion and metastasis.

\section{References}

1. Burges A and Schmalfeldt B: Ovarian cancer: diagnosis and treatment. Dtsch Arztebl Int 108: 635-641, 2011.

2. Menon U, Gentry-Maharaj A and Jacobs I: Ovarian cancer screening and mortality. JAMA 306: 1544-1545, 2011.

3. Carter JS and Downs LS Jr: Ovarian cancer tests and treatment. Female Patient (Parsippany) 36: 30-35, 2011.

4. Pejovic T and Nezhat F: Missing link: inflammation and ovarian cancer. Lancet Oncol 12: 833-834, 2011.

5. Redman C, Duffy S and Dobson C: Improving early detection of ovarian cancer. Practitioner 255: 27-30, 3, 2011.

6. Schmitt J and Matei D: Targeting angiogenesis in ovarian cancer. Cancer Treat Rev: July 14, 2011 (Epub ahead of print).

7. McCawley LJ and Matrisian LM: Tumor progression: defining the soil round the tumor seed. Curr Biol 11: R25-R27, 2001.

8. Wu B, Hu K, Li S, et al: Dihydroartiminisin inhibits the growth and metastasis of epithelial. Oncol Rep 27: 101-108, 2012.

9. Josson S, Matsuoka Y, Chung LW, Zhau HE and Wang R: Tumor-stroma co-evolution in prostate cancer progression and metastasis. Semin Cell Dev Biol 21: 26-32, 2010.

10. Anton $\mathrm{K}$ and Glod $\mathrm{J}$ : Targeting the tumor stroma in cancer therapy. Curr Pharm Biotechnol 10: 185-191, 2009.

11. Kang Y and Massague J: Epithelial-mesenchymal transitions: twist in development and metastasis. Cell 118: 277-279, 2004

12. Bacac M and Stamenkovic I: Metastatic cancer cell. Annu Rev Pathol 3: 221-247, 2008

13. Krishnamurthy S, Dong Z, Vodopyanov D, et al: Endothelial cell-initiated signaling promotes the survival and self-renewal of cancer stem cells. Cancer Res 70: 9969-9978, 2010.

14. Xouri $\mathrm{G}$ and Christian S: Origin and function of tumor stroma fibroblasts. Semin Cell Dev Biol 21: 40-46, 2010.

15. Yang J, Mani SA, Donaher JL, et al: Twist, a master regulator of morphogenesis, plays an essential role in tumor metastasis. Cell 117: 927-939, 2004.

16. Niessen K, Fu Y, Chang L, Hoodless PA, McFadden D and Karsan A: Slug is a direct Notch target required for initiation of cardiac cushion cellularization. J Cell Biol 182: 315-325, 2008.

17. Gao CF, Xie Q, Su YL, et al: Proliferation and invasion: plasticity in tumor cells. Proc Natl Acad Sci USA 102: 10528-10533, 2005.

18. Studnek J, Fernandez AR and Margolis G: When is an EMT not an EMT? JEMS 31: 36, 38-39, 2006.

19. Ikegami T, Zhang Y and Matsuzaki Y: Liver fibrosis: possible involvement of EMT. Cells Tissues Organs 185: 213-221, 2007.

20. Damonte P, Gregg JP, Borowsky AD, Keister BA and Cardiff RD: EMT tumorigenesis in the mouse mammary gland. Lab Invest 87: 1218-1226, 2007

21. Garriock RJ and Krieg PA: Wnt11-R signaling regulates a calcium sensitive EMT event essential for dorsal fin development of Xenopus. Dev Biol 304: 127-140, 2007.

22. Zhu C and Mertens PR: Epithelial-mesenchymal transition to be or not to be? Is the answer yes and no at the same time? Int Urol Nephrol 42: 843-846, 2010.

23. Yang $J$ and Weinberg RA: Epithelial-mesenchymal transition: at the crossroads of development and tumor metastasis. Dev Cell 14: 818-829, 2008.

24. Rasanen K and Vaheri A: Activation of fibroblasts in cancer stroma. Exp Cell Res 316: 2713-2722, 2010.

25. Alonso-Magdalena P, Brossner C, Reiner A, et al: A role for epithelial-mesenchymal transition in the etiology of benign prostatic hyperplasia. Proc Natl Acad Sci USA 106: 2859-2863, 2009.

26. Masuda R, Semba S, Mizuuchi E, Yanagihara K and Yokozaki H: Negative regulation of the tight junction protein tricellulin by snail-induced epithelial-mesenchymal transition in gastric carcinoma cells. Pathobiology 77: 106-113, 2010.

27. Forsyth CB, Tang Y, Shaikh M, Zhang L and Keshavarzian A: Alcohol stimulates activation of snail, epidermal growth factor receptor signaling, and biomarkers of epithelial-mesenchymal transition in colon and breast cancer cells. Alcohol Clin Exp Res 34: 19-31, 2010.

28. Hotz B, Visekruna A, Buhr HJ and Hotz HG: Beyond epithelial to mesenchymal transition: a novel role for the transcription factor Snail in inflammation and wound healing. J Gastrointest Surg 14: 388-397, 2010. 